\title{
The main-interpulse interaction of PSR B1702-19
}

\author{
P. Weltevrede ${ }^{1}$, G. A. E. Wright ${ }^{2}$, and B. W. Stappers ${ }^{3,1}$ \\ 1 Astronomical Institute "Anton Pannekoek", University of Amsterdam, Kruislaan 403, 1098 SJ Amsterdam, The Netherlands \\ e-mail: wltvrede@science.uva.nl \\ 2 Astronomy Centre, University of Sussex, Falmer, BN1 9QJ, UK \\ e-mail: G.Wright@sussex.ac.uk \\ 3 Stichting ASTRON, Postbus 2, 7990 AA Dwingeloo, The Netherlands \\ e-mail: stappers@astron.nl
}

Received 18 December 2006 / Accepted 16 February 2007

\begin{abstract}
Aims. This paper reports on single-pulse radio observations of PSR B1702-19 and their implications for pulsar emission theories. Methods. These observations were made with the Westerbork Synthesis Radio Telescope at 1380 and $328 \mathrm{MHz}$. The PA-swing is used to constrain possible geometries of the pulsar and the single-pulse data is analysed for subpulse modulation correlations between the main pulse and interpulse.

Results. We confirm earlier conclusions that the dipole axis of this pulsar is almost perpendicular to its rotation axis, and report that both its main pulse and interpulse are modulated with a periodicity around 10.4 times the pulsar's rotation. Allowing for the half-period delay between main pulse and interpulse the modulation is found to be precisely in phase. Despite small secular variations in the periodicity, the phase-locking continues over all timescales ranging up to several years.

Conclusions. The precision of the phase locking is difficult for current emission theories to explain if the main pulse and interpulse originate from opposing magnetic poles. We therefore also explore the possibility of a bidirectional model, in which all the modulated emission comes from one pole, but is seen from two sides and slightly displaced by aberration and time-delay. In this model the unmodulated emission is directed to us from the opposite pole, requiring the emission of the main pulse to originate from two different poles. This is difficult to reconcile with the observed smooth PA-swing. Whichever model turns out to be correct, the answer will have important implications for emission theories.
\end{abstract}

Key words. stars: pulsars: individual: PSR B1702-19 - stars: pulsars: general - radiation mechanisms: non-thermal

\section{Introduction}

The radio pulsar B1702-19 was discovered by Manchester et al. (1978) in the second Molonglo Survey. With a period of $0.3 \mathrm{~s}$, a characteristic age of 1.1 Myr and inferred surface magnetic field $1.1 \times 10^{12}$ Gauss, the pulsar was in no way unusual. However, some ten years later it was found to have an interpulse located some $180^{\circ}$ from the main pulse (Biggs et al. 1988), an unusual feature among pulsars, and one which has the potential for testing magnetosphere and emission models.

Radio pulsars have narrow emission beams, so that an interpulse (henceforth IP) located close to $180^{\circ}$ from the main pulse (henceforth MP) might naturally be interpreted as a view of a second magnetic pole. Previous studies of PSR B1702-19 have tended to support this view. For example, according to Biggs et al. (1988), there is little evidence for the MP-IP separation (given as $181^{\circ} \pm 1^{\circ}$ ) to evolve with frequency. Further support is given by the analyses of the shape of the polarisation position angle swing in Lyne \& Manchester (1988) and Kuzmin (1989), which is more recently confirmed by van Ommen et al. (1997). However, geometric models exist (e.g. Gil 1985; Dyks et al. 2005b) in which even a separation of this magnitude can arise from a single pole.

The structure of the MP is essentially double-peaked at all frequencies, the leading peak being more than $180^{\circ}$ from the following IP and the trailing peak less than $180^{\circ}$. The midpoint of the MP is therefore more-or-less exactly at the $180^{\circ}$ point. There is a weak shoulder on the trailing edge, so that the MP profile has been classified as triple by e.g. Rankin (1990). Karastergiou \& Johnston (2004), Gould \& Lyne (1998) and Biggs et al. (1988) also note a high degree of circular polarisation, especially at the MP. The IP itself seems to be single-peaked and almost $100 \%$ linearly polarised (e.g. Karastergiou \& Johnston 2004).

In a recent survey of pulsar modulation at an observing wavelength of $21 \mathrm{~cm}$, Weltevrede et al. (2006) found that both the MP and IP of PSR B1702-19 have apparently identical periodic intensity modulations with a periodicity $\left(P_{3}\right)$ of about $11 P$, where $P$ is the rotation period of the neutron star. This exciting result immediately suggested a method for examining interactions between the MP and IP and thereby constraining emission theories, models for subpulse drift, and magnetospheric structures.

Evidence of communication between the poles already exists in PSRs B1822-09 and B1055-52 $2^{1}$. The former is strikingly similar to PSR B1702-09, having a double-peaked MP, a weak IP $180^{\circ}$ away and a modulation with the same periodicity at both longitudes (Fowler et al. 1981; Fowler \& Wright 1982; Gil et al. 1994). However, PSR B1822-09's most remarkable feature is

${ }^{1}$ It is arguable that PSR B0950+08 also displays inter-pole communication since emission at its MP is correlated with the following IP (Hankins \& Cordes 1981). As in PSR B1055-52, the IP precedes the MP by $\sim 150^{\circ}$, but is connected to it by a clear bridge of emission. It is still a matter of debate whether this pulsar is a perpendicular rotator (Everett \& Weisberg 2001) or close to alignment (e.g. von Hoensbroech \& Xilouris 1997). 
Table 1. The details of the observations used in this paper. Here REF is the reference key used in this paper, MJD is the modified Julian date of the observation, $v$ is the central frequency of the observation, $\Delta v$ the bandwidth, $\tau_{\text {samp }}$ the sampling time, $N$ the number of recorded pulses and the last column states if the observation contains polarization information.

\begin{tabular}{ccccccc}
\hline \hline REF & MJD & $\begin{array}{c}v \\
(\mathrm{MHz})\end{array}$ & $\begin{array}{c}\Delta v \\
(\mathrm{MHz})\end{array}$ & $\begin{array}{c}\tau_{\text {samp }} \\
(\mathrm{ms})\end{array}$ & $N$ & Pol. \\
\hline 2006P & 53987 & 328 & 10 & 0.2048 & 11885 & $\mathrm{~N}$ \\
2003L & 53003 & 1380 & 80 & 0.4096 & 11071 & $\mathrm{Y}$ \\
2005L1 & 53684 & 1380 & 80 & 0.2048 & 18918 & $\mathrm{~N}$ \\
2005L2 & 53684 & 1380 & 80 & 0.2048 & 23935 & $\mathrm{~N}$ \\
\hline
\end{tabular}

the anticorrelation between the leading component of the MP and the IP, so that the pulsar frequently switches between two modes, one displaying both components of the MP, the other only the second MP component and IP emission. An apparent geometric resolution of this phenomenon has been published (Dyks et al. 2005b), but as yet lacks a physical explanation. In PSR B1822-09 the weakness of the IP has hitherto prevented any precise study of potentially revealing phase differences between the MP and IP modulations.

PSR B1055-52, like both PSRs B1702-19 and B1822-09, has a MP with a strong first component and an overlapping double second component (e.g. McCulloch et al. 1976), and again the first component appears to undergo mode-changes (Biggs 1990). But the interaction with the IP is less clear-cut: correlations of roughly one phase delay exist between the intensities of the IP and the following MP, but no comparable periodicities could be established in the modulations at either poles. Nonetheless, inter-pole communication seems to be established in both these pulsars and it represents a challenge to contemporary pulsar theory.

In this paper we set out to investigate the single-pulse behaviour of PSR B1702-19 and to establish the nature of the interactions between its MP and IP. We also try to assess the significant theoretical constraints implied by our results. In Sect. 2 our observations are listed. In Sect. 3 the profiles of both MP and IP at two frequencies are presented and a possible mode-change identified. In Sect. 4 we give a detailed analysis of the single pulse modulations in the MP and IP, and demonstrate their correlations. In Sect. 5 we use polarisation data to establish the likely viewing geometry of the observer and PSR B1702-19. Finally in Sect. 6 we suggest possible geometric configurations for the MP and IP beams and discuss their theoretical implications, before summarising our principal results in Sect. 7.

\section{Observations}

PSR B1702-19 was observed several times over three and a half years with the Westerbork Synthesis Radio Telescope (WSRT) at an observing wavelength of both 21 and $92-\mathrm{cm}$. The important parameters of the observations that are used in this paper can be found in Table 1 and each observation is given a reference key. The 2003L and the 2006P observations were part of the subpulse modulation survey done with the WSRT (Weltevrede et al. 2006, 2007). The other observations were carried out at identical radio frequencies and were reduced using the same procedure as for the survey, so for a more detailed description of the datareduction we refer to those papers.

The two 2005 observations were made directly after each other with only a few minutes between them. To obtain a high signal-to-noise $(S / N)$ profile, we summed the two integrated

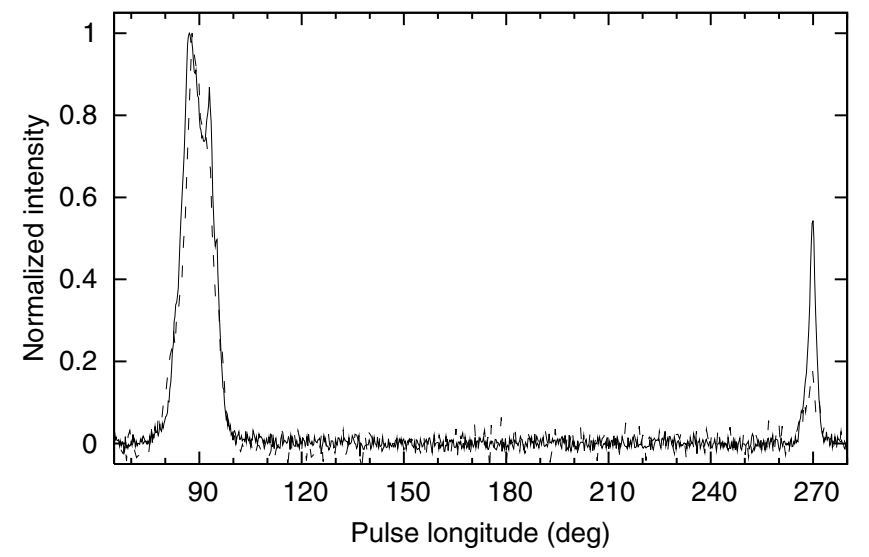

Fig. 1. The intensity profiles of the $1380 \mathrm{MHz} 2005 \mathrm{~L}$ observation (solid line) and the $328 \mathrm{MHz} 2006 \mathrm{P}$ observation (dashed line). The timeresolution of the latter is reduced by a factor two. The peak of the IP is, in both cases, put on $270^{\circ}$ pulse longitude. The two profiles are normalized to their peak intensity.

pulse profiles of the two observations together. The profiles were aligned by correlating the two profiles, which could be done with an accuracy of the sampling time $(0.2 \mathrm{~ms})$. We will use the reference key $2005 \mathrm{~L}$ for the summed profiles of the $2005 \mathrm{~L} 1$ and 2005L2 observation.

\section{The pulse profile of PSR B1702-19}

\subsection{The total intensity profile}

The pulse profiles at 1380 and $352 \mathrm{MHz}$ are shown in Fig. 1. The IP is relatively strong compared with the MP at $1380 \mathrm{MHz}$, showing that the spectra of the MP and IP are different. While the IP is single-peaked at both frequencies, the MP is double peaked, especially at high frequencies. The width of the MP does not evolve much with frequency, except that the separation between the two peaks of the MP becomes smaller at low frequencies and moreover they seem to merge.

The separation between the MP and IP is, within the noise level, the same at both frequencies and at $1380 \mathrm{MHz}$ the peak-topeak separation is $183^{\circ} \pm 0.5$. This is consistent with the absence of a frequency dependence of the MP-IP separation of $181 \pm 1^{\circ}$ reported by Biggs et al. (1988). Interestingly, the peak of the IP appears to be exactly $180^{\circ}$ from the midpoint of the two peaks of the MP. This would be in agreement with the two-pole scenario in which the magnetic axes are separated by $180^{\circ}$ and the magnetic axis coincides with the peak of the IP and the other axis with the centre of the emission cone that produces the MP. Also van Ommen et al. (1997) report a centroid MP-IP separation of $180.1 \pm 0.4^{\circ}$.

In the single wide-cone scenario (Manchester \& Lyne 1977) the MP and IP are produced by the same emission cone. In that case it would be coincidence that the component separation is very close to $180^{\circ}$. The presence of a "bridge" between the two components would be an argument in favor of a wide cone. However there is no sign for weak emission between the two components. The detection limit on the peak-flux $(3 \sigma)$ of any low-level bridge between the components is $1 \%$ of the MP intensity at $1380 \mathrm{MHz}$. There is no indication for an anti-symmetry in the shape of the MP and IP, which could have been an argument in favor of a wide-cone scenario.

Notice also that there is some indication of a third MP component at high frequencies at pulse longitude $\sim 95^{\circ}$ in Fig. 1 . 

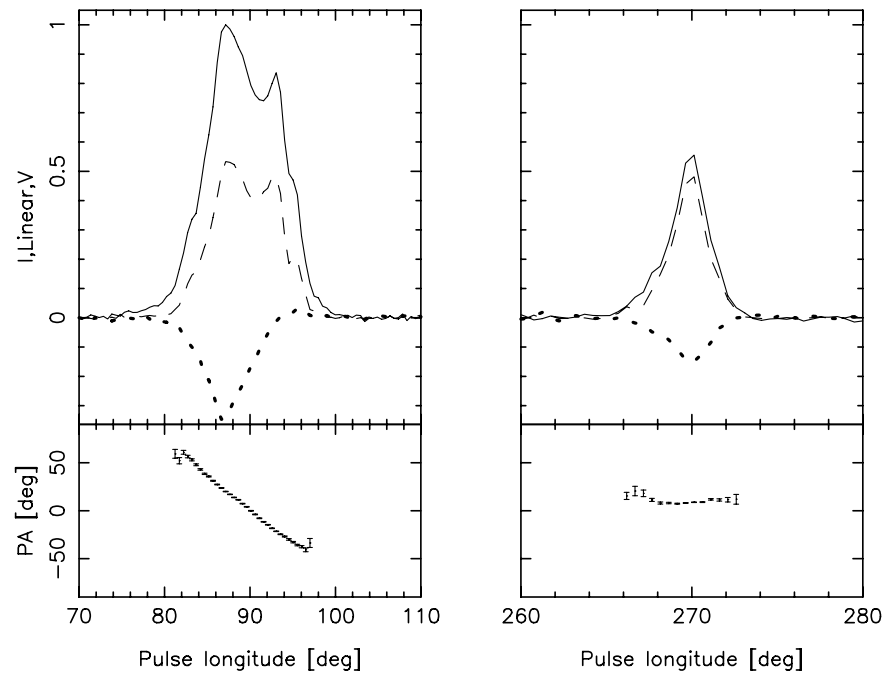

Fig. 2. The left panel shows the MP of the 1380-MHz 2003L observation and the right panel the IP. The pulse profile in total intensity (solid line), linear polarization (dashed line) and circular polarization (dotted line) are normalized to the peak intensity of the MP. The bottom panel shows the PA-swing. The pulse longitude of the peak of the IP is set to $270^{\circ}$ and at $90^{\circ}$ the PA is set to zero.

This would be consistent with the triple classification made by Rankin (1990), also noted by Gould \& Lyne (1998). Moreover, it will be shown in Sects. 3.2 and 4 that there is also evidence from the linear polarization and the pulse modulation that there is indeed an addition component at that pulse longitude.

It must be noted that there is some evidence that PSR B1702-19 occasionally changes its mode of emission, because the pulse profile published by Seiradakis et al. (1995) is significantly different from other published profiles at similar frequencies. It is striking that the MP trailing component in their $1315 \mathrm{MHz}$ profile is the strongest. Unfortunately the Effelsberg data-set does not contain single pulses, which makes it impossible to identify the possible mode-change.

\subsection{The polarization profile}

The polarization profiles of PSR B1702-19 at $1380 \mathrm{MHz}$ are shown in the top panels of Fig. 2. As one can see, the degree of linear polarization is quite high, especially in the IP. Also Karastergiou \& Johnston (2004) find that the IP is almost $100 \%$ linearly polarized at $1.41 \mathrm{GHz}$. Notice that the third component of the MP is visible in the linear polarization profile as an extra component. Also the degree of circular polarization is found to be very high for this pulsar (e.g. Gould \& Lyne 1998; Biggs et al. 1988), something that is confirmed in the WSRT data.

The emission of the MP of PSR B1702-19 is classified differently by various authors as core and conal emission. The emission of the MP is classified by Lyne \& Manchester (1988) as cone-dominated. However, it is pointed out by Karastergiou \& Johnston (2004) that the change in handedness of the circular polarization in the MP suggests that the entire MP is a core component. This in contrast to the classification made by Rankin (1990), who classified the MP as a core component with conal outriders making it a triple profile, while the IP is classified as a core single component.
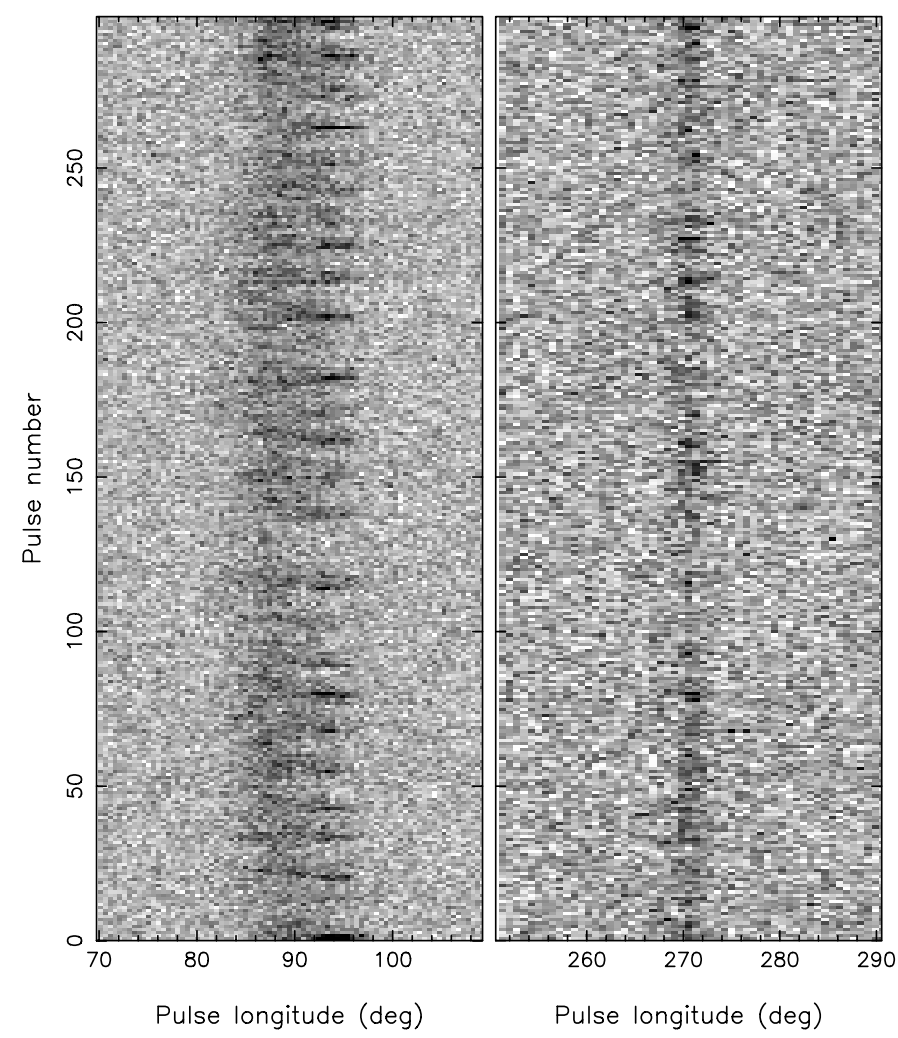

Fig. 3. This is a pulse-stack of 300 successive pulses from the 2005L1 observation. The left and right panel shows respectively the longitude range around the MP and IP. The time resolution of the left and right panels are reduced with a factor two and three respectively. Notice that the IP shows, similar to the trailing half of the MP, a $P_{3} \simeq 10 P$ modulation.

\section{Pulse modulation}

\subsection{The single pulse modulation}

It has been found by Weltevrede et al. (2006) that the single pulses of both the MP and IP show subpulse modulation and in Fig. 3 a bright piece of data is shown to illustrate this. The data in this figure is represented as a pulse-stack, i.e. the successive pulses are plotted on top of each other. The left and right panels show the single pulses of respectively the MP and IP for the same rotation of the star. The MP is clearly modulated with a periodicity of about $10 P$. The modulation is very strong in the trailing half of the MP and it resembles an on-off switching of that component with little evidence for a phase drift. A similar modulation is also just visible in the leading half of the MP and in the IP, although it is much weaker. Remarkably, the modulation of the IP appears to be more or less in phase with that of the MP (see for instance pulses 200, 210 and 220).

The presence of an intensity modulation of the single pulses is confirmed by calculating the Longitude Resolved Fluctuation Spectrum (LRFS, Backer 1970; Edwards \& Stappers 2002). The modulation is clearly detected and indeed the periodicity found in the MP and IP are very similar (Fig. 4). For the observation shown we find $P_{3}=10.4 \pm 0.3 P$, which is consistent with the result of Weltevrede et al. (2006, 2007).

Notice also that the modulation feature in the LRFS of the MP shows horizontal structure. At pulse longitude $95^{\circ}$ there is a distinct feature in the LRFS, which confirms the third emission component which was also seen in the total intensity and linear polarization profile (Fig. 2). 


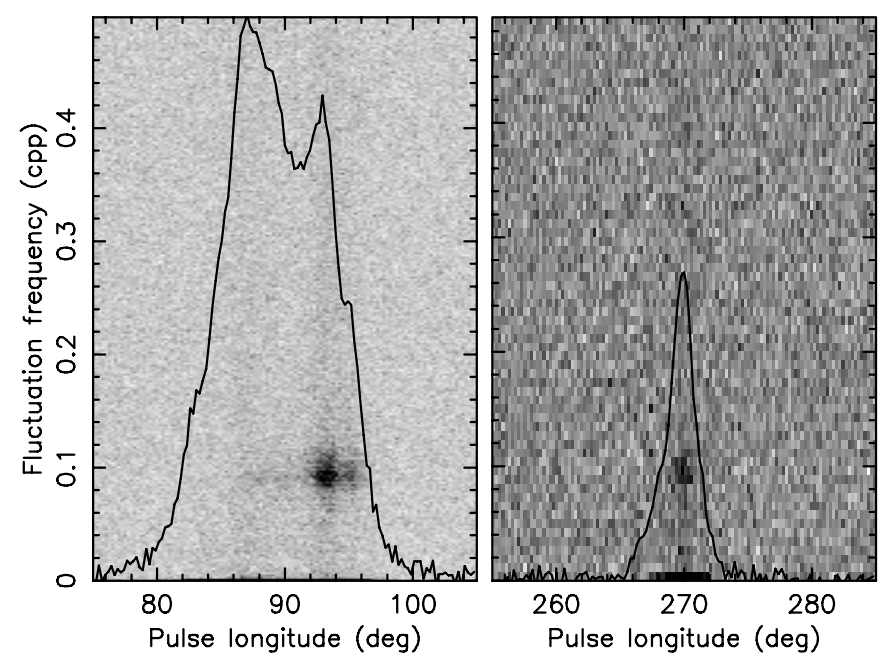

Fig. 4. The LRFS (grayscale) of the 2005L1 observation for the pulse longitude range around the MP (left) and IP (right). The vertical scale of the LRFSs are in cycles per period and the resolution is chosen differently for the MP and IP to increase the contrast. Superimposed is the shape of the pulse profile, which is normalized to have a peak intensity equal to 0.5 . Notice that both the MP and IP show modulation with the same periodicity.

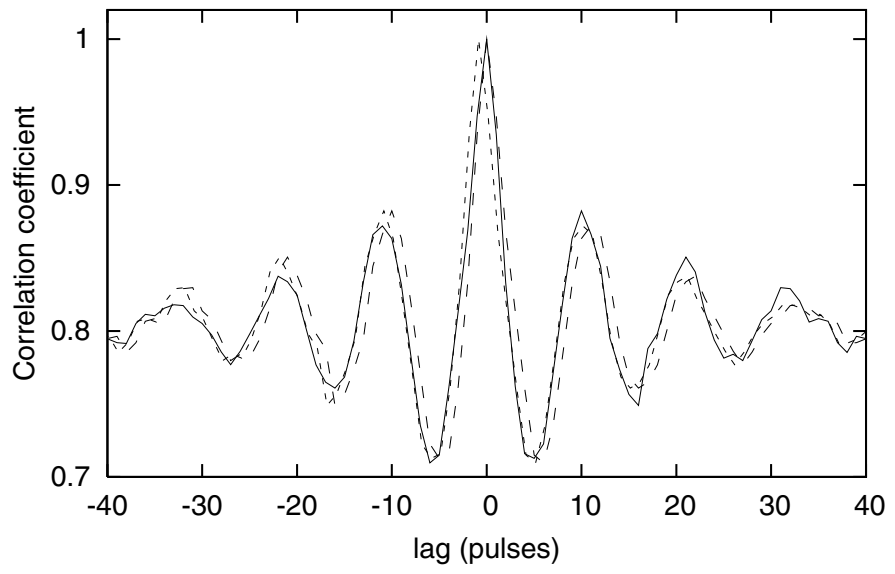

Fig. 5. The correlation between the pulse intensities of the IP and that of the trailing peak of the MP for the almost 19000 pulses of the 2005L1 observation (solid line). The correlation is normalized to the peak value and a positive lag means that the MP signal lags that of the IP. The dashed line shows the time reversed correlation and the dotted line shows the time reversed correlation that is shifted with a lag of $-0.85 P$.

\subsection{The phase locking of the modulation patterns}

Because both the MP and IP show a reasonably coherent modulation with possibly identical $P_{3}$ values there is an unique opportunity to explore the subpulse phase connection between the two components. To investigate this the single pulse intensities in the pulse longitude range of the trailing half of the MP are integrated, giving the strength of that component for each pulse. The same is done for the IP. These arrays of integrated energies of the MP and IP are correlated with each other (solid line in Fig. 5). The $P_{3} \simeq 10 P$ modulation is clearly present in the cross correlation function, which clearly confirms that both the MP and IP show a similar modulation pattern.

The periodicity in the solid line of Fig. 5 is not only seen at a delay of $\pm 10 P$, but also at higher harmonics. This is astonishing, because it demonstrates that the phase difference between the modulation pattern of the MP and IP is not significantly changed during the 19000 pulses that were recorded. So not only the $P_{3}$ value of the MP and IP are identical, but apparently the modulation patterns are phase locked over long timescales. This result could in principle be explained in two different ways:

1. the modulation of both the MP and IP are perfectly stable with strictly identical $P_{3}$ values;

2. the periodicity of the modulation patterns of the MP and IP vary slightly during the observation, but these variations are identical (and occur simultaneously) in the MP and IP.

The modulation feature in the LRFS (Fig. 4) shows vertical broadening, which means that the modulation frequency is varying slightly during the observation. This interpretation could be further supported by the absence of any correlation between the pulse energies of the MP during the first half of the observation with the pulse energies of the IP during the second half of the observation. This clearly means that the first option cannot be valid. There must be some mechanism operating in PSR B170219 that keeps the phases of the modulation patterns of the MP and IP locked.

A key result is that the correlation in Fig. 5 is not entirely symmetric under time reversal (compare the solid and dashed line). If the time reversed correlation is shifted by a lag of $-0.85 P$, it matches the solid line with high precision. This indicates that the modulation pattern of the MP lags that of the IP pulse by $0.43 P$. It can indeed be seen by eye in Fig. 3 that the modulation of the MP and IP are roughly in phase. The measured phase difference of $0.43 P$ is very close to the $0.5 P$ that is expected because the IP emission is observed half a stellar rotation later. This means that the modulation patterns emitted by the MP and IP are, correcting for this geometric delay, intrinsically in phase.

To investigate the intensity modulation in more detail, the longitude-resolved subpulse phase and the strength of the modulation is calculated for two observations (Fig. 6) using the method developed by Edwards \& Stappers (2002) and Edwards et al. (2003). The top panels confirm that the trailing half of the MP and the IP are highly modulated, while the leading component of the MP is largely unmodulated, something that can also be seen directly in the pulse-stack (Fig. 3). The 2006P observation (not plotted) also shows the modulation strongest in the trailing half of the MP, showing the similarities of the modulation at both frequencies.

The lower panels of Fig. 6 show the subpulse phase tracks of the modulation. For non-curved driftbands one expects a linear relation between the subpulse phase and the pulse longitude. For longitude-stationary subpulse modulation the subpulse phase is independent of the pulse longitude (i.e. a horizontal relation). The vertical phase scale on the left of the bottom panels is such that $360^{\circ}$ degrees corresponds to a full cycle $P_{3}$. The absolute value of the subpulse phase has no direct meaning, but differences in subpulse phase are interesting.

It can be seen in Fig. 6 that the drifting subpulse pattern for the MP has a complicated shape. In the trailing half of the MP the subpulse phase is more or less independent of pulse longitude, so the modulation is longitude stationary. However, between the peaks of the MP the subpulse phase shows an inclined track, indicating that the subpulses drift toward the leading peak of the profile. This effect is quite rapid (it takes only about $60^{\circ}$ in subpulse phase, or about $1.7 P$ before the emission drifts off the pulse window) and can therefore only just be seen by eye in the pulsestack (Fig. 3). This result confirms the presence of drifting subpulses reported by Weltevrede et al. $(2006,2007)$. Notice also 

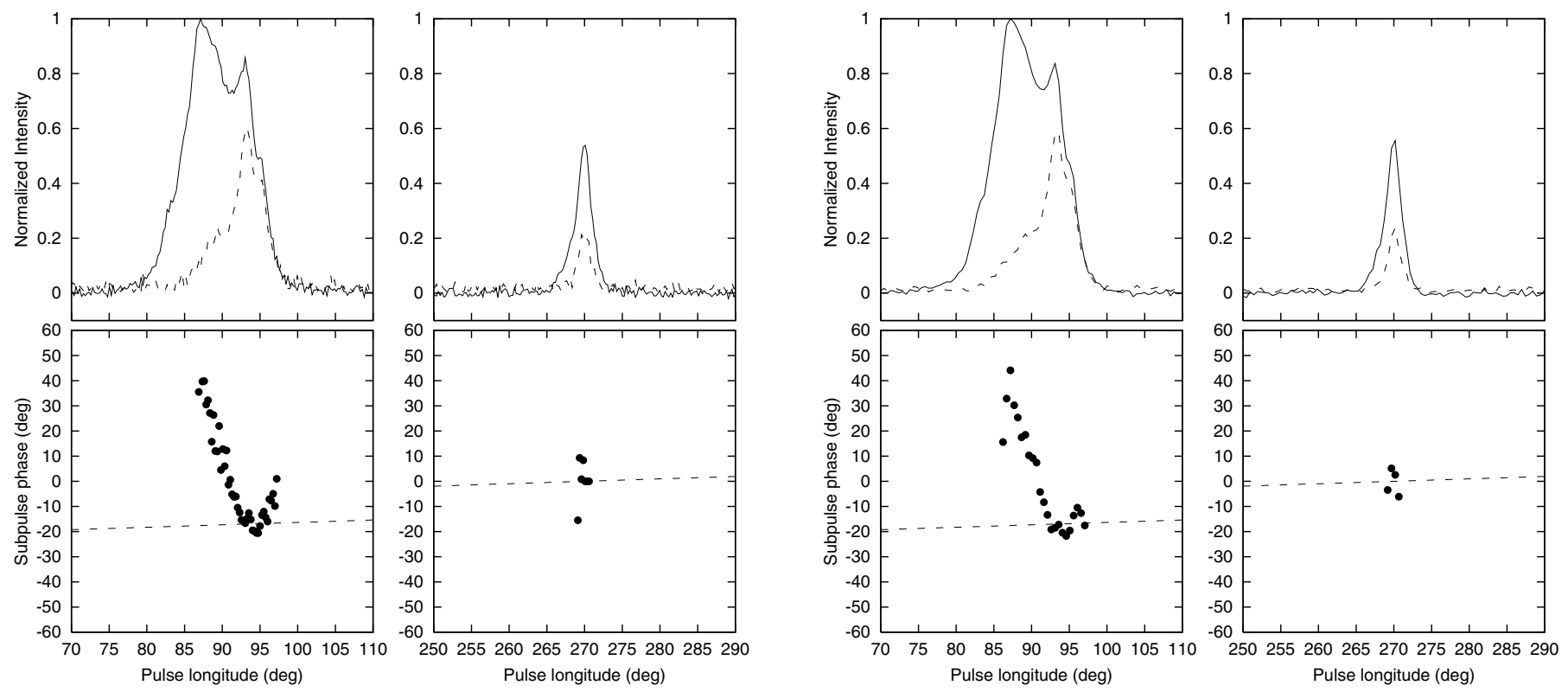

Fig. 6. The left two figures are the 2005L1 observation and the right two figures the 2003L observation. The pulse longitude ranges around the MP and IP are plotted separately. The top panels show the normalized pulse profile (solid line) and the fraction of the total intensity that is in the modulation (dashed line). The bottom panels show the longitude-resolved subpulse phase for the pulse longitudes where the modulation is detected above $3 \sigma$. The subpulse phase of the IP is set to zero. The subpulse phase at different longitudes is intrinsically in phase for points on the dashed line.

that there is some indication that subpulses also drift toward the extreme trailing edge of the MP, especially during the 2005L1 observation.

The subpulse phase of the IP is set to zero in Fig. 6. Remarkably, not only is the shape of the subpulse phase tracks very similar in the 2005 and 2003 observation, but also the phase difference between the MP and IP is, within the measurement uncertainties, identical in the two observations. This shows that the modulation patterns of the MP and IP are phase locked over a period of at least several years.

If the modulation pattern is intrinsically in phase at two pulse longitudes then we expect the observed subpulse phases to be different, because the emission is received at two different times. This geometrical delay is indicated by the dashed line in Fig. 6, which has a slope of $P / P_{3}=1 / 10.4$ degrees per degree. In both observations the modulation of the IP is intrinsically in phase with the second component of the MP, the two components of the emission which are highly modulated.

\subsection{Intensity correlation}

To further examine the degree of synchrony between the modulated emission at the MP and IP, we explored the possibility that in addition to a phase correlation between the various components of the emission, there is also an intensity correlation. To investigate this, each 11 successive pulses in the pulse-stack are summed to average out the $10.4 P$ periodicity in the intensity modulation. This is confirmed by calculating the LRFS of this sequence of integrated pulse profiles. Apart from a $\sim 5000 P$ quasi-periodicity, which is caused by the interstellar scintillation, no periodicities remain.

The cross correlations of the integrated energies between different parts of the profile are shown in Fig. 7. All the correlations show a peak at a lag of zero superimposed on a much slower decrease away from zero lag. The latter is caused by interstellar scintillation. The solid line in Fig. 7 clearly shows that there is

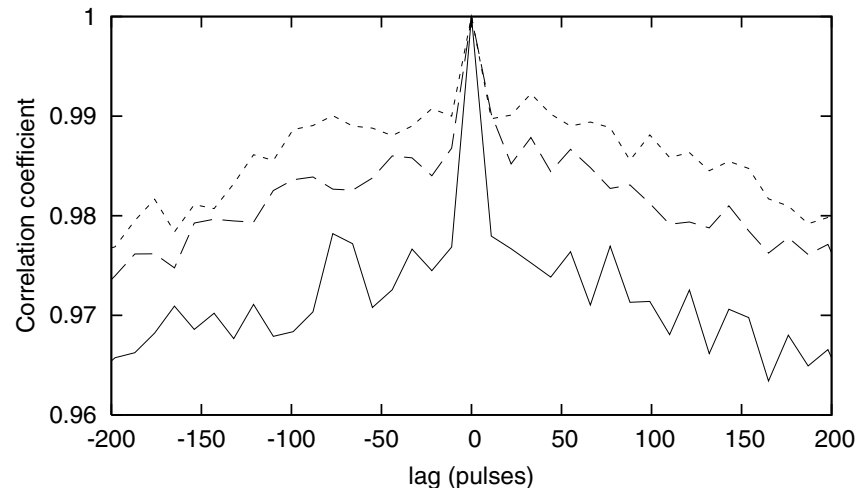

Fig. 7. The correlations between the integrated pulse energies over 11 periods of the trailing peak of the MP with the IP (solid line), leading peak of the MP with the IP (dashed line) and between both halves of the MP (dotted line). In each case the correlation coefficient is normalized to its peak value.

not only a phase lock of the modulation patterns between the trailing part of the MP with the IP, but also the intensity of the components are correlated. Notice also that the cross correlation between the two halves of the MP is weaker than the correlation between the leading half of the profile and the IP. This could be related to the fact that the proportion of the unmodulated emission in the IP is higher than that in the trailing component of the MP (see Fig. 6). If not only the intensity of the modulated part of the emission is correlated, but also the intensity variations in the unmodulated part of the emission, a stronger correlation is to be expected between the leading half of the profile and the IP than between the two halves of the MP. 

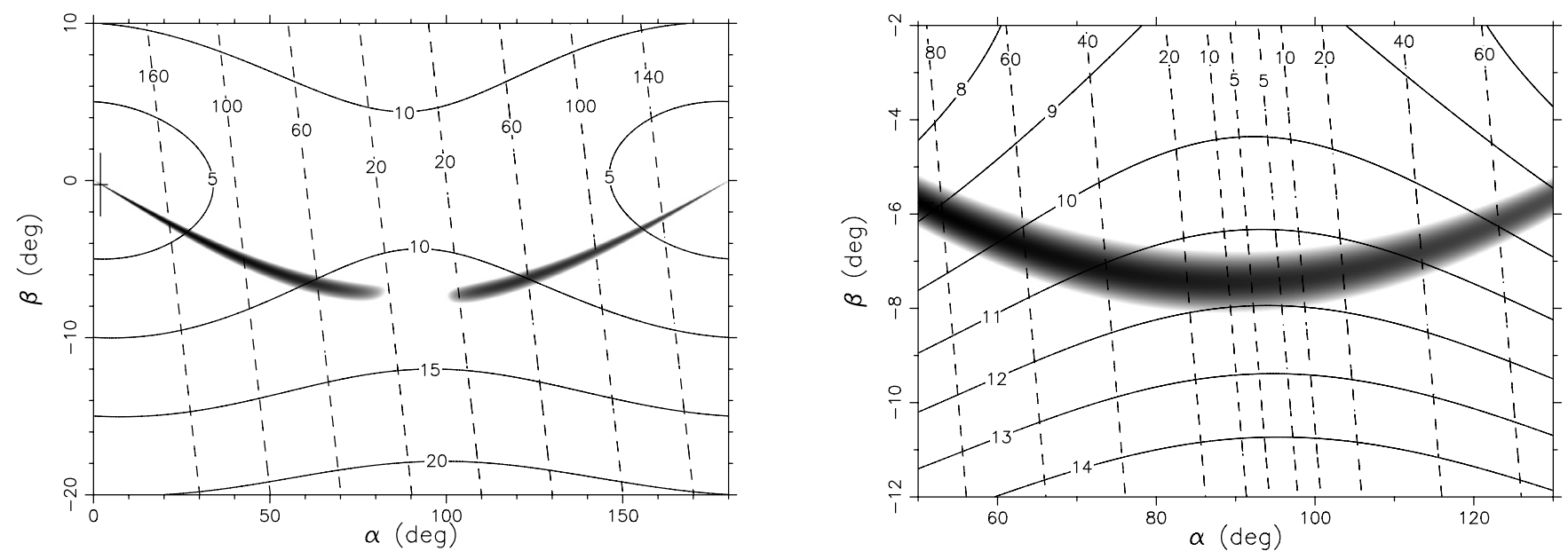

Fig. 8. Left: the $\chi^{2}$ distribution found by fitting of the entire PA-swing in the two-pole scenario. White corresponds with $\chi^{2}=15$ and black with $\chi^{2}=6$. The position of the cross (at $\alpha=1.07^{\circ}$ ) indicates the best fit. The solid and dashed contours show the derived beam radii from the pulse width of the MP and IP. Right: the right panel shows the $\chi^{2}$ distribution when only the PA-swing of the MP is fitted. The plot is zoomed in around $\alpha=90^{\circ}$.

\section{The viewing geometry}

\subsection{The PA-swing}

When the emission of the pulsar is linearly polarized the Position Angle (PA) can be measured and the PA-swing is plotted in the bottom panel of Fig. 2. The shape of PA-swings are typically S-shaped, which is interpreted in the rotating vector model (RVM; Radhakrishnan \& Cooke 1969) as the projection of dipolar fieldlines on the plane of the sky. Its precise shape depends on the orientation of the pulsar with respect to the line of sight. In particular it depends on the angle $\alpha$ between the magnetic axis and the rotation axis and the impact parameter $\beta$, which is the angle between the line of sight and magnetic axis at the position of the closest approach. For a line of sight between magnetic and rotation axis, $\beta$ is negative if $\alpha<90^{\circ}$ and positive if $\alpha>90^{\circ}$. Another angle that is often referred to in the literature is $\xi=\alpha+\beta$, which is the angle between the line of sight and the rotation axis.

The PA-swing of the MP is quite steep and smooth and can be understood in terms of the RVM. However, the PA-swing in the IP has a U-shape, something that is not expected in the RVM. Also Gould \& Lyne (1998) note that the PA of the IP has an essentially flat rotation at the higher frequencies, while it is more U-shaped at lower frequencies. It is clear that the shape of the PA-swing of the IP cannot be used to constrain the geometry. However, if the deviations from the RVM are relatively small then the average magnitude of the PA in the IP still obeys the RVM and contains useful information.

The aim in this section is to derive the geometry ( $\alpha$ and $\beta$ ) from the shape of the PA-swing. The basic approach is to do a grid search over all possible combinations of $\alpha$ and $\beta$ and for each combination the amoeba search algorithm (Press et al. 1992 ) is used to minimize the $\chi^{2}$ (which is a measure for the discrepancy between the measured and modeled PA-swing). The measurement uncertainties of the PA are taken into account in the $\chi^{2}$. The fit parameters are the pulse longitude of the magnetic axis and the PA at that position.

The fact that PSR B1702-19 has an IP is an important additional constraint to the allowed geometries. IPs can be interpreted in different ways and three different scenarios are explored in this section. One of them corresponds to a perpendicular rotator $\left(\alpha \simeq 90^{\circ}\right)$ and two to an aligned rotator $\left(\alpha \simeq 0^{\circ}\right)$.
The most straightforward interpretation (Rickett \& Lyne 1968) is a perpendicular rotator where one pole corresponds to the MP and the other to the IP. The other two scenarios involve emission from a single pole. We examine each of them in the light of our polarisation measurements.

\subsection{Two-pole model}

In the two-pole model the line of sight probes the regions near both magnetic poles $\left(\alpha \simeq 90^{\circ}\right)$. It is important to note that for the shape of the PA-swing it does not matter if we only see one or two poles. This means that the standard RVM can be used for both one and two-pole models.

In the left panel of Fig. 8 the resulting $\chi^{2}$ distribution is shown (in grayscale) for the two-pole model. The best fit has a reduced $\chi^{2} \simeq 6$. The $\chi^{2}$ distribution is basically a single arc that covers the whole $\alpha$ range, so $\alpha$ is not well constrained by the shape of the PA-swing. Solutions near $\alpha=90^{\circ}$ appear to be less favoured, which can be understood because the PA-swing of the IP is very flat and not described well by the RVM. When only the PA-swing of the MP is fitted, a $\chi^{2}$ distribution is obtained that allows solutions near $\alpha=90^{\circ}$ (right panel of Fig. 8). The PA-swing in the IP is much flatter than that of the MP and it is not even clear if the PA-swing is rising or declining in the IP. This means that the PA-swing of the IP does not constrain the fits much, except that solutions near $\alpha=90^{\circ}$ are less favoured.

The fact that the PA-swing of the IP appears to be U-shaped clearly shows that it deviates significantly from anything that would be consistent in any detail with the RVM. Nevertheless, the RVM predicts that the PA should have similar values at the longitudes of the MP and IP, consistent with the observations. Therefore it is not obvious that the flat PA-swing of the IP implies that $\alpha$ cannot be close to $90^{\circ}$. A roughly orthogonal orientation of the magnetic axis would be consistent with the reports by van Ommen et al. (1997); Lyne \& Manchester (1988); Kuzmin (1989). Gould \& Lyne (1998) find evidence that there is a $90^{\circ}$ discontinuity in the PA-swing at the leading edge of the MP, suggesting the presence of two Orthogonal Polarization Modes (OPMs). However, it seems unlikely that the MP and IP are dominated by different OPMs, because they show a similar PA.

The geometry of the system can be further constrained by considering the beam radii $\rho$ of the MP and IP. The beam radius $\rho$ 


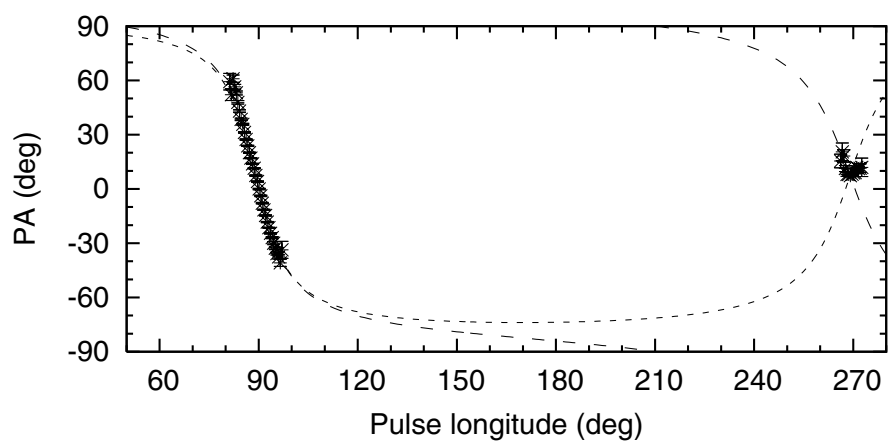

Fig. 9. The best fit of the PA-swing for the $\alpha=88^{\circ}$ and $\beta=-7.5^{\circ}$ solution (dashed line) and the $\alpha=99^{\circ}$ and $\beta=-7.5^{\circ}$ solution (dotted line). These solutions both have equal beam widths for the MP and IP $\left(\rho \simeq 12^{\circ}\right)$.

can be derived from the measured pulse width $W$ (e.g. Lorimer $\&$ Kramer 2005) and it depends on the geometry as follows:

$\cos \rho=\cos \alpha \cos \xi+\sin \alpha \sin \xi \cos \left(\frac{W}{2}\right)$.

For the IP the $W$ is the width of the IP and the sign of $\cos \alpha$ changes $^{2}$. The measured widths $W$ for the MP and IP are $18^{\circ}$ and $7^{\circ}$ respectively (the full widths as well as can be accurately determined given the $S / N$ ). The corresponding beam radii are overlaid as contours in the plots of Fig. 8. As expected in a twopole scenario $\alpha$ values close to $90^{\circ}$ are favoured because only then the beam radii of the MP and IP are similar to each other and have acceptable values.

It seems that the IP is roughly described by the RVM in the two-pole model, but is distorted by an unknown process. Therefore it makes sense to only use the MP to determine the geometry using the RVM (right panel of Fig. 8). It can be seen that for $\alpha \simeq 88^{\circ}$ and $\alpha \simeq 99^{\circ}$ the beam radii of the MP and IP are equal to each other $\left(\rho \simeq 12^{\circ}\right)$. These two solutions are plotted in Fig. 9. Both solutions describe the PA-swing of the MP equally well, but they are very different for the IP.

Assuming that the radius of the beam is set by the last open fieldline, $\rho$ can be linked to the emission height (e.g. Lorimer \& Kramer 2005) and can be approximated for $\rho \lesssim 30^{\circ}$ to

$\rho=1.24^{\circ}\left(\frac{r_{\mathrm{em}}}{10 \mathrm{~km}}\right)^{1 / 2}\left(\frac{P}{1 \mathrm{~s}}\right)^{-1 / 2}$.

Thus for a beam radius of $12^{\circ}$ and $P=0.299 \mathrm{~s}$ the emission height is $r_{\mathrm{em}}=280 \mathrm{~km}$.

\subsection{Single wide cone model}

In the single wide-cone model (Manchester \& Lyne 1977), the pulse profile is explained as a double peaked profile with a very large component separation. Such a model works best, in general, for a more aligned rotator than expected for a two-pole model. The MP and IP of PSR B1259-63 are for instance interpreted as a wide cone (Manchester \& Johnston 1995). Wide cones are especially expected for (young) pulsars with shortperiods, because their beams are wider. In this interpretation a component separation very close to $180^{\circ}$ has to be considered to

2 The geometry of the IP (indicated with primes) is related to that of the MP (without primes) as follows: $\alpha^{\prime}=180^{\circ}-\alpha, \beta^{\prime}=\beta+2 \alpha-180^{\circ}$ and $\xi^{\prime}=\xi$.

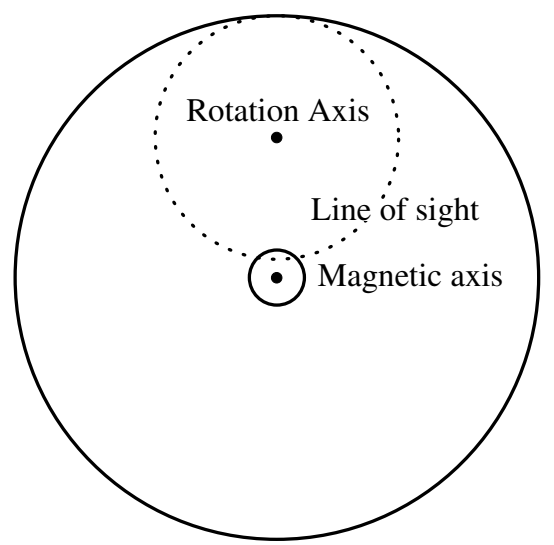

Fig. 10. The inner and outer beam interpretation for the best fit of the PA-swing with a small $\alpha$. The figure is drawn to scale. The MP is produced by the inner beam and the IP by the outer beam.

be a coincidence and the absence of a "bridge" of emission between the components also makes this interpretation less likely.

In the wide cone model the position of the magnetic axis $\left(\phi_{0}\right)$ should be located between the MP and IP. Using a grid search over all possible combinations of $\alpha$ and $\beta$ with the constraint that $\phi_{0}$ should be between pulse longitude $125^{\circ}$ and $215^{\circ}$ revealed that the lowest reduced $\chi^{2}$ found is almost 1000 . This means that the best possible fit clearly fails to describe the observed PA-swing. The reason is that the PA-swing in the MP is too steep compared with the model. A steep PA-swing implies that the magnetic axis should be close to the corresponding pulse longitude, which is not allowed in the wide cone model. Indeed, the least worst fit is found to have the magnetic pole placed right at the border of the allowed range.

Similar to the procedure for the two-cone model it is interesting to consider the possibility that the IP is in a different OPM. However, the fits turn out even worse when a jump is included in the model, because even flatter PA-swings are predicted in such a case. So in both the single wide-cone model and the twopole model it seems unlikely that the MP and IP have a different OPM.

It is clear that a single wide-cone model cannot explain the observed PA-swing, because the steepness of the PA-swing of the MP can only be explained by the RVM when the magnetic axis is close to the MP. Indeed the PA-swing of PSR B125963 , for which a wide cone is proposed (Manchester \& Johnston 1995), is much flatter.

\subsection{Inner and outer beam model}

An alternative single pole model, also for a nearly aligned rotator, is proposed by Gil (1985). This model has to be considered as a valid alternative since it also creates a frequency independent $180^{\circ}$ separation naturally. It consists of concentric inner and outer cones surrounding a single magnetic pole which is close to alignment with the rotation axis (Fig. 10). In this model either the MP or the IP component must lie where the line of sight passes close to the magnetic pole, so we can apply the results of the two-pole model (Fig. 8). The solutions with lowest $\chi^{2}$ are found for very small $\alpha$ because in this case the PA-swing in the IP is expected to be slightly flatter. The best fit has $\alpha=1.07^{\circ}$ and $\beta=-0.14^{\circ}$ and the MP is associated with the inner cone.

This interpretation could in principle also explain why the MP-IP separation is close to $180^{\circ}$ (and independent of 
frequency). However, it seems that the radius of especially the outer cone must be chosen exactly right to avoid the width of the IP becoming huge. Also, the width of the IP could be expected to be highly dependent on frequency, something that is not observed.

The beam radius of the inner cone obeys Eq. (1), while the beam radius of the outer cone can be calculated with the same equation with $\beta$ replaced by $-2 \alpha-\beta$ (and $W$ is the width of the IP $)^{3}$. This means that to explain the measured widths of the MP and IP (respectively $18^{\circ}$ and $7^{\circ}$ ) the ratio between the beam radius of the outer and inner cone for the best fit $\alpha$ and $\beta$ values is 9.5 . Note that $\beta$ is highly constrained for a given $\alpha$ such that there is a more or less linear relation between $\alpha$ and $\beta$. This means that for all choices of $\alpha$ (as long as it is small) the ratio between the inner and outer cone should also be 9.5 .

From Eq. (2) it is clear that the radius of the beam cannot be smaller than $1.24^{\circ}$, which rules out the best fit with $\alpha=1.07^{\circ}$. In the case that the inner and outer beams are produced on the same field lines, but at different heights, the emission height of the outer component should be $(9.5)^{2}$ times larger than that of the inner beam. In the extreme case that the MP is produced at the surface of the star, the emission height of the outer component should be larger than $900 \mathrm{~km}$.

Retardation and aberration will cause emission from higher in the magnetosphere to arrive earlier at the observer and a difference $\Delta h_{\mathrm{em}}$ in emission height corresponds to a pulse longitude shift of

$\Delta \phi=\frac{4 \pi \Delta h_{\mathrm{em}}}{c P}$,

where $c$ is the speed of light. A differential emission height of at least $900 \mathrm{~km}$ corresponds to a combined aberration and retardation effect of at least $7^{\circ}$ in pulse longitude, which is independent of geometry (Dyks et al. 2004). It therefore appears that even in the most extreme case the MP-IP separation could be expected to deviate strongly from $180^{\circ}$, making this model implausible. The light cylinder radius is $1.4 \times 10^{4} \mathrm{~km}$, implying that in this model the emission height of the inner cone must be less than $155 \mathrm{~km}$.

\section{Discussion}

In the preceding sections we have established the basic radio emission properties of PSR B1702-19. It is now appropriate to organise the data in an attempt to assemble a coherent model.

Two major conclusions have been reached. First, that the polarisation data is best described by a perpendicular rotator i.e. by two oppositely directed rotating vectors swept alternately through the observer's sightline. These vectors could come from opposite magnetic poles of a dipole, but we cannot rule out a priori that these two vectors are present on the same side of the pulsar, e.g. produced by upflow and downflow on the selfsame polar fieldlines at a single pole (the bidirectional model). However, this model is asymmetric and at typical emission heights the combined effects of aberration and retardation would displace their corresponding profile images from an exact $180^{\circ}$ separation.

Second, we have conclusively shown that the modulation in the MP and IP are locked into a single system, whether the pulsar's emission is examined over the long or short term. The measurable weak fluctuations in the modulation periodicity occur simultaneously at both longitudes, and this locking is maintained over tens of thousands of pulses, and even years (Figs. 5 and 6).

\footnotetext{
3 This follows directly from Fig. 10.
}
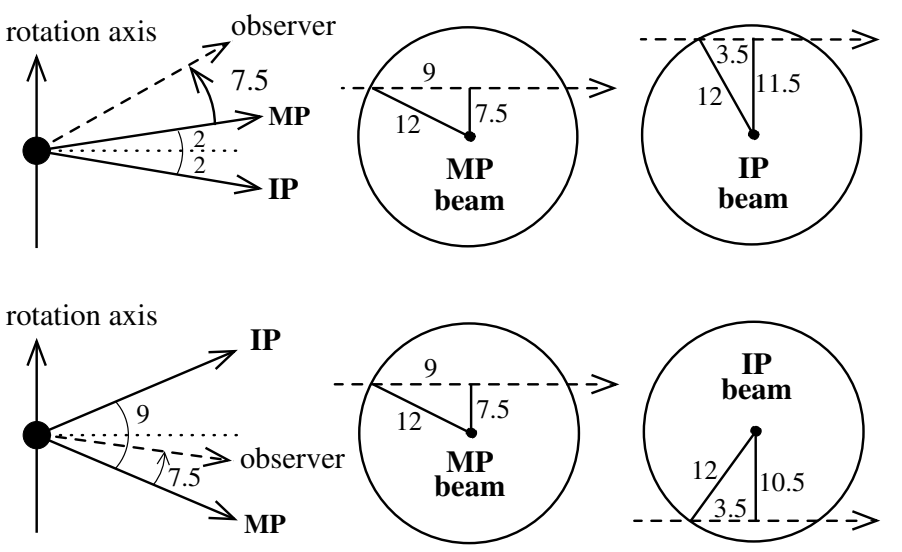

Fig. 11. The observer's MP/IP views of the pulsar's beam for the case $\alpha=88^{\circ}$ (top figures) and $\alpha=99^{\circ}$ (bottom figures). In the top figures the sightline intersects the MP and IP on the same side of the magnetic pole, but significantly separated. In the bottom figures the sightline intersections of the MP and IP beams are widely separated on opposite sides of the magnetic pole. The beam widths $\left(\rho \simeq 12^{\circ}\right)$, the measured half widths of the MP and IP (respectively $9^{\circ}$ and $3.5^{\circ}$ ) and the impact angles $\beta$ are indicated as well.

Further, we find that the intensities of the modulated emission in the MP and IP (and not only its precise repetition pattern) are correlated (Fig. 7). A final compelling argument, on the shortest timescale available, is that a cross-correlation between the trailing component of the MP and the following IP consistently yield a phase-delay in the modulations close to $0.5 \mathrm{P}$, exactly what is to be expected if both are intrinsically in phase (Figs. 5 and 6).

We will consider the implications of the two-pole and bidirectional models using only the minimum of physical assumptions. The effects of aberration and retardation are incorporated, but more complicating effects such as gravitational bending and refraction of the emission beam are ignored. This is not because we believe that these effects are unimportant, but because it is very difficult to accurately estimate them, and because it is important to establish whether a model can be constructed without their aid.

\subsection{Two-pole model}

In a model where the modulated emission is coming from two opposing poles, the simplest assumption is that PSR B1702-19 is a near-perpendicular rotating dipole and that the two poles possess an identical emission system (there is no evidence of one pole "driving" the other). One might object that the MP and IP profiles manifestly differ in shape and proportion. However, neither the observer's sightline nor the pulsar's beam is precisely perpendicular to the rotation axis and, even if the beams at both poles are identical, the observer's sightline will make differing cuts. Figure 11 shows how this may occur based on the two fits for the PA-swing from Fig. 9, which have equal beam widths. Clearly, in both cases the observer has a "fatter" view of the beam at the MP than at the IP, where the sightline merely grazes it.

Differing sightlines may therefore explain the different profiles of the MP and IP, but their observed synchrony has major implications for the emission patterns of the beams. Unless, at any given moment, the intensity of both beams is uniformly distributed, or possibly ring-like, then there is no reason to expect synchrony between two completely different sections of the 
beam. This is particularly true for the fit with $\alpha=99^{\circ}$ (bottom panel of Fig. 11), where the sightline intersects the beam on opposite sides of the magnetic pole. If one believes that the beam intensity has angular structure, whether arising from a circulating "carousel" (e.g. Ruderman \& Sutherland 1975) or varying random patches (Lyne \& Manchester 1988), it must be considered coincidental that we are able to observe two regions of the beams which are so precisely in phase with each other. This argument also applies when the MP modulation is considered alone. Figure 4 suggests a conal structure in the trailing second and third components of the MP, and that the modulation is occurring on both sides of the intersected cone. Yet Fig. 6 shows these regions to be in phase, which need not be expected from an angularly structured emission beam.

Even if we allow this phase coincidence, then the interpole synchrony is still physically difficult to understand. In the Ruderman \& Sutherland (1975) model and its successors (e.g. Gil \& Sendyk 2000; and Gil et al. 2003) the "sparking" distribution is fixed by the multipole structure on the surface of the neutron star surrounding the pole, and there is no obvious reason why these should be the same at both poles, and hence produce the same emission patterns. Even in the empirical model of Wright (2003), which requires feedback over large distances within the magnetosphere for its operation and which therefore might be expected to support inter-pole contact, it is hard to see why the system would need to arrange itself so that the poles would be in exact phase synchrony. Information travelling at the speed of light along a closed magnetic fieldline from one pole would take at the very least $0.44 P$ to reach the other (Biggs 1990). This time is far longer than the observed degree of phase locking, hence simultaneity at both poles could not be enforced in this environment. The only way events at the two poles could be kept locally simultaneous would be if information could travel through the body of the neutron star or its surface. Possible physical mechanisms for this might be oscillations of the star (e.g. Van Horn 1980, Clemens \& Rosen 2004), or variations in its net electrical charge (e.g. Michel \& Li 1999), but no detailed model based on these ideas has been worked out.

However, it should be noted that the modulation of PSR B1702-19 is not typical of that found in pulsars with coherent drifting subpulses. The modulation is dominated by intensity changes, rather than phase changes. These latter, though clearly discernible in Fig. 6 , amount to only $60^{\circ}$ of phase across the pulse window, and appear to have opposing drift directions on either side of the centroid of the trailing component of the MP. The rapid subpulse drift across the profile is also evident in Fig. 3. In the vast majority of pulsars with regular drift patterns (e.g. PSR B0809+74 which has a very similar $P_{3}$ ) the subpulses drift slowly through $360^{\circ}$ in subpulse phase in the pulse window and are mono-directional, even when the drift-mode undergoes a change. This contrasting behaviour may be due to the differing inclinations of the pulsar's magnetic axis with respect to the rotation axis. Slow-drifting pulsars such as PSR B0809+74 are often found to be relatively close to alignment $\left(\alpha<10^{\circ}\right)$ (Lyne \& Manchester 1988; Rankin 1993; Wright 2003; Rankin \& Wright 2003), while PSR B1702-19 is almost perpendicular, a factor which is likely to lead to different electric potential distributions and beam shapes close to the poles (Ruderman 1976).

The overall structure of the MP is difficult to account for. The trailing, highly modulated component, with its hint of conal structure mentioned above, is in fact only $176^{\circ}$ ahead of the IP centroid, and the role of the largely unmodulated leading component is puzzling. In the two-pole picture, the most natural explanation for the leading component would be to consider it as an integral part of the main beam, whose midpoint is separated from the IP by exactly $180^{\circ}$ at both our observing frequencies (see Fig. 1). Nothing in the smooth PA-swing across the entire MP suggests a different origin for this component, and the absence of modulation at leading longitudes, while mysterious, is also found in several pulsars with drifting emission (Weltevrede et al. 2006, 2007). It is however strange that its intensity is so weakly coupled with that of the modulated component over the $P_{3}$ cycle (Fig. 7) and appears to be virtually decoupled from the pulsar's modulation cycle. It might be possible to claim that this component represents "core" emission, which is supported by the presence of strong circular polarisation which often accompanies such a feature (Rankin 1990). However, core components in highly-inclined pulsars are normally narrow and are thought by some (Rankin 1990) to be formed close to the neutron star surface. In any case, core components should, by definition, form close to the magnetic axis, and there is nothing in the RVM fits to suggest that our sightline passes close to the axis. Perhaps the best that can be said is that the component does not have any apparent conal characteristics.

\subsection{Bidirectional emission}

It has recently been suggested (Dyks et al. 2005b) that at least some of the MP emission in pulsars with IPs of nearly $180^{\circ}$ separation from the MP may be directed downwards towards the neutron star and viewed from the opposite side as an IP. This radical idea may be usefully applied to PSR B1702-19, since it offers a natural way to explain the synchrony of the trailing component of the MP and the IP by supposing that the source of the modulated emission is viewed twice per stellar rotation. Whatever the physical nature of the source, it would seem at a stroke to explain why the modulation is exactly in phase over short and long timescales. However, as with the two-pole model of the previous section, it does not explain why the structure of the emission beam (Fig. 11) is so tightly in phase, and we must assume that, whether viewed from front or back, the emission must have a ring or uniform structure within the beam.

The resulting geometry of this bidirectional model differs in significant points of detail from that of Dyks et al. (2005b), since these authors associate different components of the pulse profile with the reversible source in the pulsar they analyse (PSR B1822-09, see next section). But it shares with that model a fundamental difference to the classical two-pole model of Sect. 6.1, in that viewing a rotating vector at a fixed difference above the stellar surface will inevitably entail aberration and retardation effects, causing the viewer to see the back view of the vector slightly later than $180^{\circ}$ from its front view.

If we assume the midpoint of the MP occurs at the minimum between its two components, then the bidirectional model cannot be correct. This is because this point is precisely $180^{\circ}$ from the centroid of the IP, so it would imply no aberrational of retardation effects. However, if we take an alternative view of the MP based on the pulsar's subpulse behaviour rather than its integrated profile we come to a different picture. Disregarding for the moment the unmodulated leading component of the MP and focus on the phase-locked modulated components, then we notice first of all that the modulated second component of the MP is clearly an overlapping double component (Fig. 4), which are seen to be in phase-lock with the IP (Fig. 6). Therefore the natural choice for the centroid of the modulated second component of the MP would be the midpoint between the two modulated regions, which would be the centre of the postulated conal ring of modulated emission. 

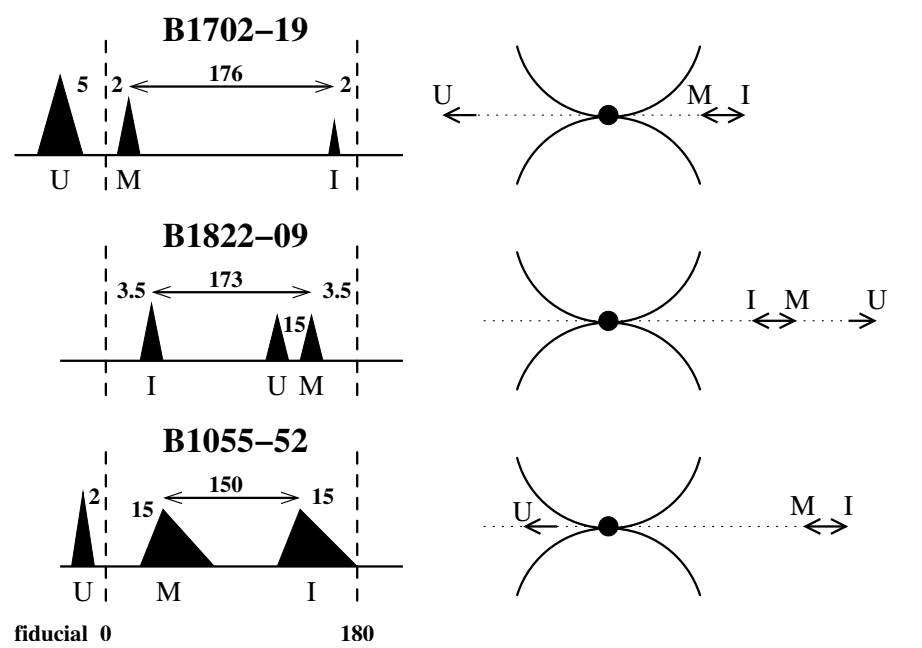

Fig. 12. The relative phase separations (in degrees) of PSRs B170219, B1822-09 and B1055-52, and their translations into a bidirectional model. The fiducial points of each refers to the derived longitude of the magnetic poles on the star at the instant they are directed to the observer. Both the phase plots (left) and dipolar models (right) are indicative and not to scale. The IP, the modulated and unmodulated components are indicated by respectively I, M and $\mathrm{U}$ (see text for estimated heights).

This point is $176^{\circ}$ ahead of the IP, a shift of $4^{\circ}$ from the MP's emission minimum. If this point and the IP are to be considered as sightline sections across reverse images of one another, then the sense of the shift is such that the IP beam must be directed to us from the front of the star, and the MP's second component is an emission beam from behind the star (Fig. 12). To take into account retardation and aberration (but neglecting gravitational bending and refraction) in a bidirectional model we may use Eq. (3) with the differential height $\Delta h$ now replaced by $2 h_{\mathrm{em}}$, where $h_{\mathrm{em}}$ is the distance of the source from the neutron star (Dyks et al. 2004, 2005b).

$\Delta \phi=\frac{8 \pi h_{\mathrm{em}}}{c P}$.

Setting $\Delta \phi=4^{\circ}$, we obtain an emission height of $250 \mathrm{~km}$ for the source of the "I/M" source, roughly consistent with our estimate of the beam emission height in Sect. 5.2. Such an emission height is not incompatible with he wide range of estimates that observers and theorists have made for pulsar emission heights over the years, although it tends to be at the lower end.

We now need to explain the origin of the leading component of the MP, and this is best done with reference to the fiducial points marked in Fig. 12 (upper diagram). These indicate the pulse longitudes at which the magnetic poles on the surface of the star are directed to the observer. The peak of the leading component of the MP appears about $7^{\circ}$ in advance of the modulated component of the MP, which means that the unmodulated component "U" must point towards us about $5^{\circ}$ of longitude ahead of the fiducial point on the star, giving it an altitude of some $600 \mathrm{~km}$ (Eq. (3) with $\Delta h_{\mathrm{em}}$ replaced by $h_{\mathrm{em}}$ ). The emission beam would have a radius of $\rho=18^{\circ}$ at this altitude if it is confined by the last closed fieldlines (Eq. (2)), which corresponds to an expected component width of $W=32^{\circ}$ (Eq. (1)). The observed width of the unmodulated component is clearly narrower (Fig. 2), showing that the model requires the beam corresponding to this component to be considerably narrower than the cone defined by the last closed fieldlines. This may not be an insuperable problem, since there is evidence that some pulsars indeed have beams which lie well within the last open fieldlines (Gangadhara \& Gupta 2001; Gupta \& Gangadhara 2003; Mitra \& Rankin 2002).

However attractive the bidirectional model may appear, significant objections remain. Firstly, the model is asymmetric why don't we see modulated emission from the second pole? Secondly, and perhaps more seriously, the PA-swing from the leading to trailing component of the MP is very smooth (Fig. 2). If the two components originate from opposite sides of the star, aberrational and time-delay effects would shift their PAswings out of phase (Dyks et al. 2005a; Blaskiewicz et al. 1991). Possibly, the PA of the unmodulated emission dominates the polarisation picture for the MP, although this cannot explain the IP emission, which is highly modulated and is almost $100 \%$ linearly polarised. Thirdly, this model is purely geometric in nature and needs to explain how coherent radiation of the same frequency is emitted at roughly the same height. Upflow and downflow would need to be simultaneously present, perhaps resulting from modulated pair-creation in the magnetosphere.

\subsection{Comparisons with other interpulse pulsars}

\subsubsection{PSR B1822-09}

PSR B1822-09 offers an immediate comparison in many ways. Like PSR B1702-19, this pulsar has a double-peak MP and an IP approximately $180^{\circ}$ away. Furthermore, its MP's trailing component and IP are both periodically modulated with the same $P_{3}(\approx 40 P)$ (Fowler et al. 1981). It is not known whether these components are closely phase-locked, as in PSR B1702-19, but if this were to prove so, then many of our above arguments in favour of an annular emission beam and a possible common source for the modulated components might apply to this pulsar also.

It is possible to construct a bidirectional model for PSR B1822-09 along the same lines as PSR B1702-19 (Fig. 12). However, there are significant differences in the component separations (Gil et al. 1994), and this gives the model a different structure. Firstly, in PSR B1822-09 the IP follows both the components of the MP by more than $180^{\circ}$. When we apply the logic of PSR B1702-19's model to PSR B1822-09 and insist that the two modulated components are emitted from the same vector, then the IP must be the beam directed toward the star. Secondly, the unmodulated leading component would point towards the observer ahead of the common emission region for the MP and IP (see Fig. 12).

The period of PSR B1822-09 is 0.77 seconds, more than twice that of PSR B1702-19, and the derived height for the I/M source is about $1100 \mathrm{~km}$ (Eq. (4)) and the leading component $6000 \mathrm{~km}$ (Eq. (3) with $\Delta h_{\mathrm{em}}$ replaced by $h_{\mathrm{em}}$ ). Note from Fig. 12 that although the arrangement of the components for this pulsar is somewhat different - in that we seem to only see the emission from one side of the star - the U component is again considerably farther from the star than the I/M region and must therefore be narrower about the magnetic axis than the periodically modulated region.

The model described above is somewhat different then the Dyks et al. (2005b) model, because they interpret the modechanges of PSR B1822-09 as reversals of the emission beam. PSR B1822-09 either shows a strong leading MP component, or a strong IP and therefore they associate the IP with the leading " $U$ " component of the MP, rather than with the "M" component. If refraction and gravitational bending can be neglected, 
the "I/U" should be located about $3500 \mathrm{~km}$ above the surface of the star.

\subsubsection{PSR B1055-52}

Quite apart from having an IP, this interesting pulsar has many similarities to PSR B1702-19. It has the same inferred surface field strength $\left(1.1 \times 10^{12}\right.$ Gauss $)$ and a period and timing age of just half that of PSR B1702-19 (500 kyr and 0.20 s). Furthermore, the form of its MP, like PSR B1702-19, consists of a strong leading component and an overlapping double second component. Biggs (1990) even reports the detection of a weak modulation feature of $P_{3}=10 P$ in the second component of the MP, despite his single pulse data having a weak $S / N$. PSR B1055-52 is also known to be a powerful source of X-rays (it is one of the three Musketeers; e.g. Cheng \& Helfand 1983; De Luca et al. 2005), which could ultimately lead to a better understanding of its geometry.

As in PSR B1822-09, and possibly PSR B1702-19, the leading component of PSR B1055-52's MP seems to be implicated in a mode-change (see Fig. 12 of Biggs 1990). The pulsar's weak $S / N$ hitherto prevented a more detailed single-pulse analysis, so the exact implications of the mode-change for the other components and intensity modulations are unknown.

If we assume that the modulated component of the MP and IP share a common source, the model for PSR B1702-19 might apply to this pulsar also. The structure and relative positioning of the MP and IP follow PSR B1702-19 in having less than $180^{\circ}$ between them, so a bidirectional model based on their separations results in the same relative pattern for their components (Fig. 12). The "M" component is placed at the location of the minimum in the middle of the "conal" trailing component of the MP and the "I" component is taken to be the minimum in the middle of the IP component (see Fig. 11 of Biggs 1990). These component separations would imply an emission height of $1300 \mathrm{~km}$ for the bidirectional vector and an emission height of only $150 \mathrm{~km}$ for the unmodulated source. It should be noted that the widths of the MP and IP of PSR B1055-52 are considerably larger than that of PSRs B1702-19 and B1822-09, and therefore the precise pulse longitudes of the " $U$ ", "M" and "I" peaks are less clearly defined.

\section{Summary}

We have investigated the properties of PSR B1702-19. The pulsar is unusual in having both a main pulse (MP) and an interpulse (IP). Here we give a summary of our results.

Both the MP and the IP are modulated with a period of $P_{3}=10.4 \pm 0.3 P$, comprising an intensity modulation with only weak phase drift. There is a phase difference close to $0.5 P$ between the modulation of the trailing component in the MP and that in the IP, demonstrating that these two regions of the pulsar are intrinsically exactly in phase. This phase lock is held over many modulation cycles, despite small secular changes in the periodicity. There is no evidence from observations over widely separated epochs that the modulations ever fall out of phase. Even when integrated over the emission cycle, the intensities of the IP and the highly-modulated trailing component of the MP are correlated.

It is difficult to explain the phase synchrony with "carousel" or "patchy" beams. It would have to be a coincidence that our traverses of the MP and IP beams intersect regions which are exactly in phase. This would suggest a beam structure with a modulated uniform cone of emission, contrary to the expectations of much current theory.

The polarisation data implies that the magnetic axis of PSR B1702-19 rotates almost perpendicularly to its rotation axis, which means that models with a single or double emission cones close to alignment can be eliminated.

If the modulated emission in the MP and IP come from two different magnetic poles, then to keep them in the observed synchrony only models with virtually instantaneous communication between the poles are possible. These may involve processes which have attracted little theoretical attention in the past, such as non-radial oscillations of the star.

If the modulated emission is assumed to be oppositely directed on the same fieldline on the same side of the star, it seems possible to construct a model which accounts for the slight deviations of these components from $180^{\circ}$ separation in terms of aberration and retardation. It would mean the IP emission is directed upwards and the modulated component of the MP directed downwards and observed from behind the star. The unmodulated emission from the MP would be directed upwards on the opposite magnetic pole to the sources of the modulated emission. However, such a model requires the emission of the MP to originate from two different poles, which is difficult to reconcile with the observed smooth PA-swing. Whether the bidirectional or the two-pole model turns out to be correct, the answer will have important implications for emission theories.

There is a striking similarity between PSRs B1702-19 and B1822-09, a pulsar which also shows periodic modulations at the MP and IP. If a phase lock operates between them as strictly as in PSR B1702-19, then the same problems of interpole communication will apply.

Acknowledgements. We would like to express our gratitude to J. H. Seiradakis and A. Jessner, who kindly made available archival Effelsberg data and helped us with the data reduction. G.A.E.W. thanks The Netherlands Foundation for Scientific Research (NWO) and the Anton Pannekoek Institute, Amsterdam, for their kind hospitality and the University of Sussex for a Visiting Fellowship. The Westerbork Synthesis Radio Telescope is operated by the ASTRON (Netherlands Foundation for Research in Astronomy) with support from NWO.

\section{References}

Backer, D. C. 1970, Nature, 227, 692

Biggs, J. D. 1990, MNRAS, 246, 341

Biggs, J. D., Lyne, A. G., Hamilton, P. A., McCulloch, P. M., \& Manchester, R. N. 1988, MNRAS, 235, 255

Blaskiewicz, M., Cordes, J. M., \& Wasserman, I. 1991, ApJ, 370, 643

Cheng, A. F., \& Helfand, D. J. 1983, ApJ, 271, 271

Clemens, J. C., \& Rosen, R. 2004, ApJ, 609, 340

De Luca, A., Caraveo, P. A., Mereghetti, S., Negroni, M., \& Bignami, G. F. 2005, ApJ, 623, 1051

Dyks, J., Rudak, B., \& Harding, A. K. 2004, ApJ, 607, 939

Dyks, J., Frąckowiak, M., Słowikowska, A., Rudak, B., \& Zhang, B. 2005a, ApJ, 633, 1101

Dyks, J., Zhang, B., \& Gil, J. 2005b, ApJ, 626, L45

Edwards, R. T., \& Stappers, B. W. 2002, A\&A, 393, 733

Edwards, R. T., Stappers, B. W., \& van Leeuwen, A. G. J. 2003, A\&A, 402, 321

Everett, J. E., \& Weisberg, J. M. 2001, ApJ, 553, 341

Fowler, L. A., Morris, D., \& Wright, G. A. E. 1981, A\&A, 93, 54

Fowler, L. A., \& Wright, G. A. E. 1982, A\&A, 109, 279

Gangadhara, R. T., \& Gupta, Y. 2001, ApJ, 555, 31

Gil, J. 1985, ApJ, 299, 154

Gil, J. A., \& Sendyk, M. 2000, ApJ, 541, 351

Gil, J. A., Jessner, A., Kijak, J., et al. 1994, A\&A, 282, 45

Gil, J., Melikidze, G. I., \& Geppert, U. 2003, A\&A, 407, 315

Gould, D. M., \& Lyne, A. G. 1998, MNRAS, 301, 235

Gupta, Y., \& Gangadhara, R. T. 2003, ApJ, 584, 418

Hankins, T. H., \& Cordes, J. M. 1981, ApJ, 249, 241

Karastergiou, A., \& Johnston, S. 2004, MNRAS, 352, 689

Kuzmin, A. D. 1989, Sov. Astron. Lett., 15, 189 
Lorimer, D. R. \& Kramer, M. 2005, Handbook of Pulsar Astronomy (Cambridge University Press)

Lyne, A. G., \& Manchester, R. N. 1988, MNRAS, 234, 477

Manchester, R. N., \& Johnston, S. 1995, ApJ, 441, L65

Manchester, R. N., \& Lyne, A. G. 1977, MNRAS, 181, 761

Manchester, R. N., Lyne, A. G., Taylor, J. H., et al. 1978, MNRAS, 185, 409

McCulloch, P. M., Hamilton, P. A., Ables, J. G., \& Komesaroff, M. M. 1976, MNRAS, 175, 71P

Michel, F. C., \& Li, H. 1999, Phys. Rep., 318, 227

Mitra, D., \& Rankin, J. M. 2002, ApJ, 577, 322

Press, W. H., Teukolsky, S. A., Vetterling, W. T., \& Flannery, B. P. 1992, Numerical recipes in $\mathrm{C}$. The art of scientific computing 2nd edn. (Cambridge: University Press)

Radhakrishnan, V., \& Cooke, D. J. 1969, ApJL, 3, 225
Rankin, J. M. 1990, ApJ, 352, 247

Rankin, J. M. 1993, Ap\&SS, 85, 145

Rankin, J. M., \& Wright, G. A. E. 2003, A\&ARv, 12, 43

Rickett, B. J., \& Lyne, A. G. 1968, Nature, 218, 934

Ruderman, M. 1976, ApJ, 203, 206

Ruderman, M. A., \& Sutherland, P. G. 1975, ApJ, 196, 51

Seiradakis, J. H., Gil, J. A., Graham, D. A., et al. 1995, Ap\&SS, 111, 205

Van Horn, H. M. 1980, ApJ, 236, 899

van Ommen, T. D., D’Alesssandro, F. D., Hamilton, P. A., \& McCulloch, P. M. 1997, MNRAS, 287, 307

von Hoensbroech, A., \& Xilouris, K. M. 1997, A\&AS, 126, 121

Weltevrede, P., Edwards, R. T., \& Stappers, B. W. 2006, A\&A, 445, 243

Weltevrede, P., , Stappers, B. W., \& Edwards, R. T. 2007, A\&A, submitted Wright, G. A. E. 2003, MNRAS, 344, 1041 